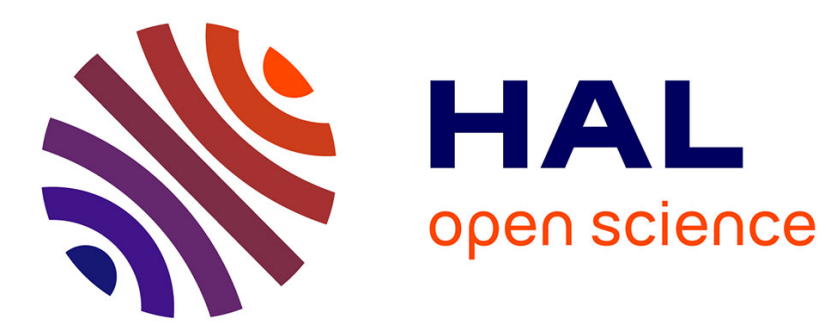

\title{
Collapse of a flexible polymer chain II
}

P.-G. de Gennes

\section{To cite this version:}

P.-G. de Gennes. Collapse of a flexible polymer chain II. Journal de Physique Lettres, 1978, 39 (17), pp.299-301. 10.1051/jphyslet:019780039017029900 . jpa-00231502

\section{HAL Id: jpa-00231502 https://hal.science/jpa-00231502}

Submitted on 1 Jan 1978

HAL is a multi-disciplinary open access archive for the deposit and dissemination of scientific research documents, whether they are published or not. The documents may come from teaching and research institutions in France or abroad, or from public or private research centers.
L'archive ouverte pluridisciplinaire HAL, est destinée au dépôt et à la diffusion de documents scientifiques de niveau recherche, publiés ou non, émanant des établissements d'enseignement et de recherche français ou étrangers, des laboratoires publics ou privés. 
Classification

Physics Abstracts

$61.40 \mathrm{~K}-64.70$

\title{
COLLAPSE OF A FLEXIBLE POLYMER CHAIN II
}

\author{
P. G. DE GENNES
}

\author{
Collège de France, place M.-Berthelot, 75231 Paris Cedex 05, France
}

(Reçu le 12 mai 1978, accepté le 12 juillet 1978)

\begin{abstract}
Résumé. - On essaie de construire ici une description physique de la transition subie par une chaîne unique, longue ( $N$ monomères), et flexible, au point $\Theta$ de Flory, à partir des résultats de la littérature sur un point tricritique en dimension 3. On trouve que pour $T=\Theta+\Delta T$, la chaîne se comporte comme une séquence de blobs, chacun avec un certain nombre $G_{\mathrm{B}}$ de monomères$$
G_{\mathrm{B}} \sim(\Theta / \Delta T)^{2} \ln |\Theta / \Delta T|^{-3 / 11} .
$$

A l'intérieur de chaque blob les conditions $\Theta$ sont remplies. Mais à plus grande échelle, le collier de blobs montre, soit un comportement de volume exclu $(T>\Theta)$, soit un comportement effondré avec $R \sim N^{1 / 3}(T<\Theta)$. La partie singulière de l'énergie libre est $\sim k T$ par blob, d'où une anomalie de chaleur spécifique $C \sim(\ln \Theta / \Delta T)^{3 / 1}$. Plutôt que les mesures thermiques, une étude de l'hypochromisme en spectroscopie UV permettrait peut-être de tester ce point.
\end{abstract}

Abstract. - This note gives a physical description of the collapse transition for a long chain ( $N$ monomers) near the $\Theta$ point, based on existing renormalization group calculations.

1) at $T=\Theta$ exactly, the chain is ideal as regards the size $\left(R \sim N^{1 / 2}\right)$ and the virial coefficient vanishes. However, some repulsive correlations remain due to 3 body interactions ;

2) for temperatures $T=\Theta+\Delta T$, a sufficiently long chain can be pictured as a sequence of blobs, each with $G_{\mathrm{B}}$ monomers $G_{\mathrm{B}}=\left(\frac{\Theta}{\Delta T}\right)^{2}\left(\ln \left|\frac{\Theta}{\Delta T}\right|\right)^{-3 / 11}$. Inside each blob, the $\Theta$ conditions still hold. But on a larger scale, the string of blobs shows excluded volume behaviour (if $T>\Theta$ ) or collapse, with $R \sim N^{1 / 3}$ (if $T<\Theta$ ). The singular part of the free energy is of order $k T$ per blob : this gives a specific heat divergence of $\left(\ln \left|\frac{\Theta}{\Delta T}\right|\right)^{3 / 11}$. A better probe could be obtained with UV spectra, measuring the number of monomer-monomer contacts.

1. Introduction. - The conformations of a single chain near the Flory compensation point [1] are extremely delicate to handle. Numerical data on lattices suggest a specific heat anomaly [2]. The problem is related to a tricritical point [3] and is particularly difficult in 3 dimensions, where logarithmic singularities occur [4]. Tricritical behaviour is understood in the language of field theory [5] or parquet diagrams [6]. The translation of these results into polymer physics has been faced with difficulties [7]. Here we use a relatively simple approach based on a decimation on the chemical sequence [8].

In the Flory language [1], the free energy per site on the lattice, as a function of the density $\Phi$, has the form :

$$
\left.\frac{F}{k T}\right|_{\text {site }}=\frac{1}{2} u \Phi^{2}+\frac{1}{6} t \Phi^{3}+\cdots
$$

where

$$
u=1-2 \chi \cong \frac{\Delta T}{\Theta}
$$

is small, while $t$ is positive and finite. The monomer size is a constant $a$. The three numbers $a, u, t$ specify the problem entirely.

In the decimation method, we group the monomers into subunits of $g$ monomers, and calculate the new parameters $a_{1}, u_{1}, t_{1}$ for the subunits. We then iterate the process $m$ times. The resulting trajectories in the $u, t$ plane are shown on figure 1 . If we start from $\mathrm{A}$, we go towards a fixed point $u=u^{*}, t=0$. This means that large subunits repel each other like hard spheres [8] and give an excluded volume behaviour. If we start from $B$, we go towards large negative values of $u=$ large subunits tend to collapse. If we start from a point $\mathrm{C}$ on the dividing line, we go towards the origin (ideal chain behaviour). The dividing line defines a certain $u$ for each $t$, or a certain $\Theta$ point for each $t$. (In the original Flory picture, where all renormalizations of $\Theta$ are ignored, the dividing line is vertical : we shall also assume this, in what follows, since it simplifies the notation.) 


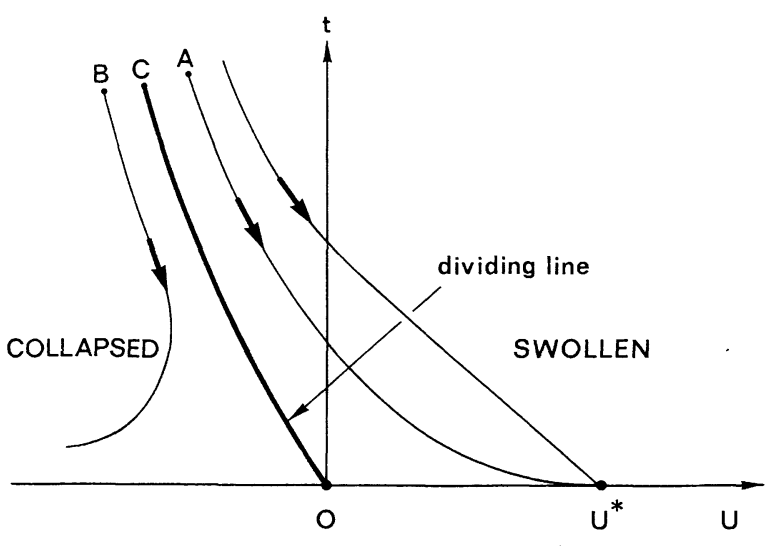

FIG. 1.

2. Chain behaviour at $T=\Theta$ exactly. - Near the origin, the recurrence equations can be obtained by perturbation theory. On the dividing line, they reduce to $\left({ }^{1}\right)$

$$
\begin{aligned}
a_{m+1} & =a_{m} g^{1 / 2}\left(1+Q_{1} t_{m}^{2}\right) \\
t_{m+1} & =t_{m}\left(1-Q_{2} t_{m}\right) .
\end{aligned}
$$

In equation (2a) the factor $g^{1 / 2}$ corresponds to ideal chain behaviour; the linear corrections $\left(u_{m}\right.$ and $\left.t_{m}\right)$ cancel out on the dividing line (by definition). In equation $(2 b)$ the coefficient of $t_{m}$ is unity : ideal subunits of volume $a_{1}^{3}=g^{3 / 2} a^{3}$ have a 3-body interaction $\operatorname{tg}^{3}\left(a / a_{1}\right)^{6} \equiv t$. From equation (2) we find a slow decrease :

$$
t_{m}=\frac{Q_{3}}{m} \quad(m \gg 1) .
$$

However for sufficiently large subunits, $t_{m}$ (and $u_{m}$ ) vanish : a dilute solution is ideal for $T=\Theta$. Inserting equation (3) into equation (2a) and integrating we get :

$$
a_{m}=a g^{m / 2}\left(1+\frac{Q_{4}}{m}\right) .
$$

Choosing $m$ such that one subunit of the final stage spans the whole chain $\left(g^{m / 2}=N\right)$, we see that the size $R$ of the chain has the form first calculated by Stephen [9]

$$
R=a N^{1 / 2}\left(1+\frac{Q_{5}}{\ln N}\right) .
$$

In practice this correction is negligible and $R \sim N^{1 / 2}$. This result depends crucially on the fact that the corrections in equation (2a) are only of order $t^{2}$. From it one can also show that the pair correlation function at the $\Theta$ point is ideal $\langle\rho(0) \rho(r)\rangle \sim 1 / r$.

( $\left.{ }^{1}\right) Q_{1}, Q_{2}, \ldots$ are numerical constants; some of them depend on the choice of $g$, but the final results (as in eq. (5)) do not.
3. Vicinity of the $\Theta$ point : the blob picture. - The 3-body interaction $t$ will turn out to be more important when we look at the approach of the $\Theta$ point : we now start at a point $\mathrm{A}\left(u_{0}, t_{0}\right)$ just off the dividing line, and write the recurrence equation for $u$

$$
u_{m+1}=u_{m} g^{1 / 2}\left(1-Q_{6} t_{m}\right)+\mathrm{O}\left(u_{m}^{2}\right) .
$$

After renormalization of the $\Theta$ point, terms like $t_{m}$ or $t_{m}^{2}$ cannot appear on the right hand side of (6). If $u_{m}=0$, we must stay on the dividing line and $u_{m+1} \equiv 0$.

Let us assume first that $t_{m}$ is still given by (3), and integrate, obtaining :

$$
\begin{aligned}
u_{m} & =u_{0} g^{m / 2} m^{-p} \\
p & =Q_{3} Q_{6}=4 / 11
\end{aligned}
$$

where we have inserted the exact value of $p$ taken from reference [5]. Physically $u_{m}$ is the analog of the $\zeta$ parameter introduced in perturbation expansions [10] : if we use subunits of $G$ monomers $\left(G=g^{m}\right)$ we have

$$
\zeta \cong G^{1 / 2} u_{0} /(\ln G)^{p} .
$$

The correlations at the $\Theta$ point have reduced $\zeta$ by a factor $(\ln G)^{-p}$.

Let us now return to the equation for $t_{m}$ : off the dividing line it becomes instead of (2)

$$
\begin{aligned}
\frac{\partial t}{\partial m}=t_{m+1}-t_{m}=-Q_{2} & t_{m}^{2}+ \\
& +Q_{7} u_{m}^{2}+Q_{8} u_{m} t_{m} .
\end{aligned}
$$

(There are no linear terms in $u$ : in first order a two point interaction cannot generate a three point interaction.) The dominant correction can be checked to be $u_{m}^{2}$. Using (7), it gives a shift in $t_{m}$ of value

$$
\delta t_{m} \sim u_{0}^{2} g^{m} m^{-2 p}
$$

This will be negligible when compared to (3) provided that

$$
u_{0}^{2} \approx g^{-m} \cdot m^{2 p-1} .
$$

This allows us to define a blob ( $G_{\mathrm{B}}$ monomers) : we impose that the inequality (10) be equivalent to $g^{m}<G_{\mathrm{B}}$. Then we know that $\Theta$ point singularities are dominant inside a blob, but are negligible between blobs. (For $g^{m}>G_{\mathrm{B}}$ we move off the dividing line.) From (10) we then have

$$
G_{\mathrm{B}}=u_{0}^{-2}\left|\ln u_{0}\right|^{-(1-2 p)}
$$

with $u_{0} \sim \Theta /|\Delta T|$. Equation (12) is the key to the physics of the $\Theta$ region. From equation (5), each blob has a size $r_{\mathrm{B}}=G_{\mathrm{B}}^{1 / 2} a$. 
4. The many blob regime. - For a fixed chain length $N$ we have two regimes :

a) Inner $\Theta$ regime: $G_{\mathrm{B}}>N$, very near $u_{0}=0$.

b) Outer $\Theta$ regime : $G_{\mathrm{B}}<N$, for slightly larger $\left|u_{0}\right|$. We shall be concerned now with the second case, assuming $1 \ll G_{\mathrm{B}} \ll N$. Consider first the size $R$ of the chain for $u_{0}>0$. The blobs have a two body interaction

$$
u_{\mathrm{B}}=u_{0} G_{\mathrm{B}}^{1 / 2}\left|\ln u_{0}\right|^{-p} \cong\left|\ln u_{0}\right|^{-1 / 2}
$$

and the three body interaction between blobs is irrelevant here. We can then apply the Flory formula to the string of blobs [1], and obtain :

$$
\begin{aligned}
R^{5} & =r_{\mathrm{B}}^{5} u_{\mathrm{B}}\left(\frac{N}{G_{\mathrm{B}}}\right)^{3} \\
R & =N^{3 / 5} a\left[\frac{\Theta}{\Delta T}\left(\ln \frac{\Theta}{\Delta T}\right)^{p}\right]^{1 / 5} .
\end{aligned}
$$

Consider now the poor solvent side : here the analog of equation (1) is now written in terms of a number $\rho$ of blobs per volume $r_{\mathrm{B}}^{3}$

$$
\frac{1}{k T} F_{\mathrm{int}}=\frac{1}{2}\left|u_{\mathrm{B}}\right| \rho^{2}+\frac{1}{6} t_{\mathrm{B}} \rho^{3}
$$

where $t_{\mathrm{B}} \cong\left(\ln \frac{\Theta}{\Delta T}\right)^{-1}$ from equation (3). The opti- mum $\rho$ is $\left|u_{\mathrm{B}}\right| / t_{\mathrm{B}}$ and this defines the size $R$ through $\rho\left(R / r_{\mathrm{B}}\right)^{3}=N / G_{\mathrm{B}},[12]$

$$
R^{3} \cong N a^{3} \frac{\Theta}{\Delta T}\left[\ln \frac{\Theta}{\Delta T}\right]^{-7 / 11}
$$

Note that equations $(14,15)$ do not crossover into $R=\left(N^{1 / 2} a\right)$ when $G_{\mathrm{B}}=N$, but rather when $G_{\mathrm{B}} \cong N / \ln N$. For instance, when $u_{0}>0$, the interval $N / \ln N<G_{\mathrm{B}}<N$ corresponds to blobs between which excluded volume effects are weak.

Let us end up with the chain free energy $F$. From the diagram

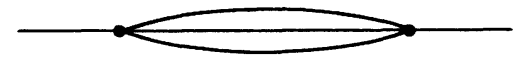

we are led to a contribution of order

$$
u_{\mathrm{B}}^{2} \int_{a}^{r_{\mathrm{B}}} \frac{1}{r_{12}^{3}} \mathrm{dr}_{12} \sim u_{\mathrm{B}}^{2}\left|\ln u_{0}\right| \sim 1
$$

for $F / k T$ per blob. This then gives

$$
\frac{F_{\mathrm{tot}}}{k T}=\frac{N}{G_{\mathrm{B}}} \sim N\left(\frac{\Delta T}{\Theta}\right)^{2}\left(\ln \left|\frac{\Theta}{\Delta T}\right|\right)^{1-2 p}
$$

and a specific heat peak with a $\ln ^{3 / 11}$ power. This differs slightly from the proposal of Moore [7]. Rather than thermal measurements, a study of hypochromism in UV spectroscopy would measure the number of binary contacts $N_{\mathrm{c}} \sim \partial F / \partial u_{0}$ inside the chain. We then expect a singularity in $\partial N_{\mathrm{c}} / \partial T \sim \ln ^{3 / 11}$ which might be interesting to see.

References

[1] FloRY, P., Principles of Polymer chemistry (Cornell University Press, Ithaca N.Y.).

[2] Domb, C., Polymer 15 (1974) 259 ;

Rapaport, D. C., Phys. Lett. 48A (1974) 339; J. Phys. A 10 (1977) 637.

[3] De Gennes, P. G., J. Physique Lett. 36 (1975) L-55.

[4] See for instance : Toulouse, G., Pfeuty, P., Introduction au groupe de renormalisation (Presses Univ. Grenoble), 1975.

[5] Riedel, E., Wegner, F., Phys. Rev. B 7 (1973) 248. WOHRER, M., Thèse $3^{\mathrm{e}}$ cycle, Université Paris VI (1976).

[6] Abrahams, E., Stephen, M., Straley, J. P., Phys. Rev. B 12 (1975) 256

[7] Oono, Y., Oyama, T., J. Phys. Soc. Jpn 44 (1978) 301 ; For an earlier attempt see MOoRE, M., J. Phys. A 10 (1977) 305.
The main difference between the present work and this paper lies in the logarithmic renormalization of $u$ described by equation (7).

[8] De Gennes, P. G., Nuovo Cimento 7 (1977) 363.

[9] STEPHEN, M., Phys. Lett. 53A (1975) 363.

[10] Yamakawa, H., Modern theory of polymer solutions, Harper and Row (N. Y.), 1971.

[11] In earlier work, omitting all logarithmic singularities, I suggested the approximate form $r_{\mathrm{B}} \cong a u_{0}^{-1}$ which is in reasonable agreement with the neutron data : see FARNoux, B. et al., J. Physique 39 (1978) 77

[12] Evidence for $R^{3} \sim N$ can be found in neutron data on small chains : Nierlich, M., Cotton, J. P., Farnoux, B., - J. Chem. Phys., to be published. 